Research Report Poster Presentation

Number: RR-PO-08-18-Sat

Saturday 2 May 2015 13:00

Exhibit halls 401-403

\section{IMPORTANCE OF PROGRAM PHYSICAL ACTIVITY IN QUALITY OF LIFE IN MULTIPLE SCLEROSIS PATIENTS, SIX MONTHS AFTER THE YOUR INTERVENTION}

$\underline{\text { L. Pedro }}^{1}$, J. Pais-Ribeiro ${ }^{2}$,

J. Pascoa Pinheiro ${ }^{3}$

${ }^{1}$ Politecnic of Lisbon - ESTeSL, Physical Therapy, Lisbon,

Portugal; ${ }^{2}$ University of Porto, FPCE, Porto,

Portugal; ${ }^{3}$ University of Coimbra, Medicine, Coimbra,

Portugal

Background: Multiple Sclerosis (MS) is a chronic disease of the central nervous system that affects more often young adults in the prime of his career and personal development, with no cure and unknown causes. The most common signs and symptoms are fatigue, muscle weakness, changes in sensation, ataxia, changes in balance, gait difficulties, memory difficulties, cognitive impairment and difficulties in problem solving MS is a relatively common neurological disorder in which various impairments and disabilities impact strongly on function and daily life activities.

Purpose: The aim of this study is to examine the implications of an Intervention Program of Physical Activity (IPPA) in quality of life in MS patients, six months after the intervention.

Methods: This is a quasi experimental study, the hypothesis is: people with MS that practice physical activity have a better the quality of life six months after de intervention program. The study includes a consecutive sample of $27 \mathrm{MS}$ patients, diagnosed at least one year, and with an Expanded Disability Status Scale (EDSS) score under seven. The sample was divided in three groups, each group including eight people, collected sequentially in three Portuguese hospitals (Lisbon, Coimbra, and Porto) with the collaboration of their personal physician. The sessions were held once a week for 90 minutes, over a period of seven weeks. We used the Quality of Life Questionnaire, Quality of Life in Multiple Sclerosis - MSQOL - 54, at the beginning of the intervention program and six months after the end of the program.

The IPPA objective is to promote autonomous physical activity visa a better quality of life. Each session aimed: to stimulate group discussion about a theme related to inability or limitation in physical activity; to discuss strategies to minimize these limitations; to define and learn appropriate physical activities to implement between sessions.

Results: The age range of the subjects was between 20 and 58 years with a mean age of 44 years. $58.3 \%$ were women, $37.5 \%$ were currently married, $67 \%$ were retired and the mean level of education was 12.5 years.
We use non parametric test, related-samples Wilcoxon signed ranks test to compare the quality of life between the beginning of the intervention program (Time A) and six months after the end of the program (time $\mathrm{C}$ ).

There were statistically significant differences between Time A and Time C, the dimensions of MSQOL-54: Overall quality of life, Role physical limitation, body pain and health perception, with positive results in Time $\mathrm{C}$.

The results suggest that this intervention program based on the implementation of individual strategies to increase physical activity, helps to improved your physical performance, control pain, increase the perception of health and quality of life in patients of multiple sclerosis.

Conclusion(s): This intervention program to promote physical activity, based on the facilitation of personal implementation strategies, can be very beneficial in promoting quality of life in people with multiple sclerosis.

Implications: The Development of a physical therapy program, with purpose promote autonomous physical activity and quality of life in patients of multiple sclerosis.

Keywords: Physical activity; Multiple sclerosis; Quality of life

Funding acknowledgements: Work was unfunded.

Ethics approval: The work follows the recommendations of the Declaration of Helsínquia.

http://dx.doi.org/10.1016/j.physio.2015.03.2108

Research Report Poster Presentation

Number: RR-PO-12-24-Sat

Saturday 2 May 2015 13:00

Exhibit halls 401-403

\section{EXPLORING WORK-RELATED SPINAL CORD INJURIES IN GAUTENG PROVINCE, SOUTH AFRICA}

\author{
N. Pefile $^{1}$, S. Visagie ${ }^{2}$, S. Gcaza ${ }^{2}$ \\ ${ }^{1}$ University of Kwazulu-Natal, Physiotherapy, Durban, \\ South Africa; ${ }^{2}$ Stellenbosch University, Centre for \\ Rehabilitation Studies, Cape Town, South Africa
}

Background: Spinal cord injury (SCI) is devastating and an impact negatively on an individual's functioning and social participation and they are preventable. Before any preventative programme can be embarked upon, stakeholders must understand the current situation.

Purpose: Therefore the aim of the study was to describe work-related spinal cord injuries in Gauteng Province, South Africa.

Methods: This was a descriptive, retrospective study in which mixed methods were used. The research was completed in two private rehabilitation units in Gauteng Province, South Africa. During the first phase 135 medical files/records of persons admitted between 1999-2009, who had suffered work-related SCIs, were perused. No sampling was done. 